\title{
Density by Moduli and Lacunary Statistical Convergence
}

\author{
Vinod K. Bhardwaj ${ }^{1}$ and Shweta Dhawan ${ }^{2}$ \\ ${ }^{1}$ Department of Mathematics, Kurukshetra University, Kurukshetra 136119, India \\ ${ }^{2}$ Department of Mathematics, KVA DAV College for Women, Karnal 132001, India \\ Correspondence should be addressed to Vinod K. Bhardwaj; vinodk_bhj@rediffmail.com
}

Received 15 November 2015; Accepted 5 January 2016

Academic Editor: Feyzi Başar

Copyright (c) 2016 V. K. Bhardwaj and S. Dhawan. This is an open access article distributed under the Creative Commons Attribution License, which permits unrestricted use, distribution, and reproduction in any medium, provided the original work is properly cited.

\begin{abstract}
We have introduced and studied a new concept of $f$-lacunary statistical convergence, where $f$ is an unbounded modulus. It is shown that, under certain conditions on a modulus $f$, the concepts of lacunary strong convergence with respect to a modulus $f$ and $f$ lacunary statistical convergence are equivalent on bounded sequences. We further characterize those $\theta$ for which $S_{\theta}^{f}=S^{f}$, where $S_{\theta}^{f}$ and $S^{f}$ denote the sets of all $f$-lacunary statistically convergent sequences and $f$-statistically convergent sequences, respectively. A general description of inclusion between two arbitrary lacunary methods of $f$-statistical convergence is given. Finally, we give an $S_{\theta}^{f}$-analog of the Cauchy criterion for convergence and a Tauberian theorem for $S_{\theta}^{f}$-convergence is also proved.
\end{abstract}

\section{Introduction and Historical Background}

Statistical convergence is a generalization of the usual notion of convergence. The idea of statistical convergence was given in the first edition (published in Warsaw in 1935) of the monograph of Zygmund [1], who called it "almost convergence." Formally the concept of statistical convergence was introduced by Fast [2] and Steinhaus [3] independently in the year 1951 and later reintroduced by Schoenberg [4] in the year 1959 .

Although statistical convergence was introduced over nearly the last sixty years, it has become an active area of research in recent years. Statistical convergence has been studied by several authors [5-14].

Let $\mathbb{N}$ denote the set of all natural numbers. A number sequence $x=\left(x_{k}\right)$ is said to be statistically convergent to the number $l$ if for each $\varepsilon>0$ the set $\left\{k \in \mathbb{N}:\left|x_{k}-l\right| \geq \varepsilon\right\}$ has natural density zero, where the natural density of a subset $K \subset \mathbb{N}$ (chapter 11 , see [15]) is defined by

$$
d(K)=\lim _{n \rightarrow \infty} \frac{1}{n}|\{k \leq n: k \in K\}|,
$$

where $|\{k \leq n: k \in K\}|$ denotes the number of elements of $K$ not exceeding $n$. Obviously we have $d(K)=0$ provided that $K$ is a finite set of positive integers. If a sequence is statistically convergent to $l$, then we write it as $S-\lim x_{k}=l$ or $x_{k} \rightarrow l(S)$. The set of all statistically convergent sequences is denoted by $S$.

The notion of modulus function was introduced by Nakano [16] in 1953. Following Ruckle [17] and Maddox [18], we recall that a modulus $f$ is a function from $[0, \infty)$ to $[0, \infty)$ such that

(i) $f(x)=0$ if and only if $x=0$,

(ii) $f(x+y) \leq f(x)+f(y)$ for $x \geq 0, y \geq 0$,

(iii) $f$ is increasing,

(iv) $f$ is continuous from the right at 0 .

Because of (ii), $|f(x)-f(y)| \leq f(|x-y|)$ so that, in view of (iv), $f$ is continuous everywhere on $[0, \infty)$. A modulus may be unbounded or bounded. For example, $f(x)=x^{p}$, where $0<p \leq 1$, is unbounded, but $f(x)=x /(1+x)$ is bounded.

Connor [11], Öztürk and Bilgin [19], Ghosh and Srivastava [20], Bhardwaj and Singh [21-23], Çolak [24], Altin and Et [25], Aizpuru et al. [5], Bhardwaj and Dhawan [6], Bhardwaj et al. [26], and some others have used a modulus function to construct some sequence spaces.

In the year 2014, Aizpuru et al. [5] defined a new concept of density with the help of an unbounded modulus function 
and, as a consequence, they obtained a new concept of nonmatrix convergence, namely, $f$-statistical convergence, which is intermediate between the ordinary convergence and the statistical convergence and agrees with the statistical convergence when the modulus function is the identity mapping.

Quite recently, Bhardwaj and Dhawan [6] and Bhardwaj et al. [26] have introduced and studied the concepts of $f$ statistical convergence of order $\alpha$ and $f$-statistical boundedness, respectively, by using the approach of Aizpuru et al. [5].

We now recall some definitions that will be needed in the sequel.

Definition 1 (see [5]). Let $f$ be an unbounded modulus function. The $f$-density of a set $A \subset \mathbb{N}$ is defined by

$$
d^{f}(A)=\lim _{n \rightarrow \infty} \frac{f(|\{k \leq n: k \in A\}|)}{f(n)}
$$

in case this limit exists. Clearly, finite sets have zero $f$-density and $d^{f}(\mathbb{N}-A)=1-d^{f}(A)$ does not hold, in general. But if $d^{f}(A)=0$ then $d^{f}(\mathbb{N}-A)=1$. Note that Aizpuru et al. [5] have used the notation $d_{f}(A)$ to denote the $f$-density of a set A.

Remark 2. For any unbounded modulus $f$ and $A \subset \mathbb{N}$, $d^{f}(A)=0$ implies that $d(A)=0$. But converse need not be true in the sense that a set having zero natural density may have nonzero $f$-density with respect to some unbounded modulus $f$. For example, if we take $f(x)=\log (x+1)$ and $A=\{1,4,9, \ldots\}$, then $d(A)=0$ but $d^{f}(A)=1 / 2$.

Definition 3 (see [5]). Let $f$ be an unbounded modulus function. A number sequence $x=\left(x_{k}\right)$ is said to be $f$ statistically convergent to $l$ or $S^{f}$-convergent to $l$, if, for each $\varepsilon>0$,

$$
\begin{array}{r}
d^{f}\left(\left\{k \in \mathbb{N}:\left|x_{k}-l\right| \geq \varepsilon\right\}\right)=0, \\
\text { that is, } \lim _{n \rightarrow \infty} \frac{1}{f(n)} f\left(\left|\left\{k \leq n:\left|x_{k}-l\right| \geq \varepsilon\right\}\right|\right)=0,
\end{array}
$$

and one writes it as $S^{f}-\lim x_{k}=l$ or $x_{k} \rightarrow l\left(S^{f}\right)$. The set of all $f$-statistically convergent sequences is denoted by $S^{f}$.

In view of Definition 3 and Remark 2, it follows that every $f$-statistically convergent sequence is statistically convergent, but a statistically convergent sequence need not be $f$ statistically convergent for every unbounded modulus $f$.

By a lacunary sequence $\theta=\left(k_{r}\right), r=0,1,2, \ldots$, where $k_{o}=0$, we will mean an increasing sequence of nonnegative integers with $k_{r}-k_{r-1} \rightarrow \infty$ as $r \rightarrow \infty$. The intervals determined by $\theta$ will be denoted by $I_{r}=\left(k_{r-1}, k_{r}\right]$, and we let $h_{r}=k_{r}-k_{r-1}$. The ratio $k_{r} / k_{r-1}$, which also occurs frequently, will be denoted by $q_{r}$.

The space of all lacunary strongly convergent sequences, $N_{\theta}$, was defined by Freedman et al. [27] as follows:

$$
\begin{aligned}
& N_{\theta}=\left\{x=\left(x_{k}\right):\right. \\
& \left.\lim _{r \rightarrow \infty} \frac{1}{h_{r}} \sum_{k \in I_{r}}\left|x_{k}-l\right|=0 \text { for some number } l\right\} .
\end{aligned}
$$

If $x$ is lacunary strongly convergent to $l$, then we write $N_{\theta}-$ $\lim x_{k}=l$ or $x_{k} \rightarrow l\left(N_{\theta}\right)$.

There is a strong connection [27] between $N_{\theta}$ and the space $w$ of strongly Cesàro summable sequences, which is defined by

$$
\begin{aligned}
w= & \left\{x=\left(x_{k}\right):\right. \\
& \left.\lim _{n \rightarrow \infty} \frac{1}{n} \sum_{k=1}^{n}\left|x_{k}-l\right|=0 \text { for some number } l\right\} .
\end{aligned}
$$

In the special case, where $\theta=\left(2^{r}\right)$, we have $N_{\theta}=w$. In fact, for a lacunary sequence $\theta, N_{\theta}=w$ if and only if $1<$ $\lim \inf _{r} q_{r} \leq \lim \sup _{r} q_{r}<\infty$ ([27], on page 511).

Note that the space $w$ of strongly Cesàro summable sequences was denoted by $\left|\sigma_{1}\right|$ in [27].

In the year 1986, Maddox [28] extended the concept of strong Cesàro summability to that of strong Cesàro summability with respect to a modulus $f$. A sequence $x=$ $\left(x_{k}\right)$ is said to be strongly Cesàro summable with respect to a modulus $f$ to $l$ if

$$
\lim _{n \rightarrow \infty} \frac{1}{n} \sum_{k=1}^{n} f\left(\left|x_{k}-l\right|\right)=0 .
$$

The space of strongly Cesàro summable sequences, with respect to a modulus $f$, is denoted by $w(f)$.

Further, in the year 1994, the notion of lacunary strong convergence was extended to that of lacunary strong convergence with respect to a modulus $f$ by Pehlivan and Fisher [29]. The space $N_{\theta}(f)$ of lacunary strongly convergent sequences with respect to a modulus $f$ is defined as

$$
\begin{aligned}
& N_{\theta}(f)=\left\{x=\left(x_{k}\right): \lim _{r \rightarrow \infty} \frac{1}{h_{r}} \sum_{k \in I_{r}} f\left(\left|x_{k}-l\right|\right)\right. \\
& =0 \text { for some number } l\} .
\end{aligned}
$$

If $x$ is lacunary strongly convergent with respect to a modulus $f$ to $l$, then we write $N_{\theta}(f)-\lim x_{k}$ or $x_{k} \rightarrow l\left(N_{\theta}(f)\right)$.

Remark 4. We will denote the spaces $w(f)$ and $N_{\theta}(f)$ by $w^{f}$ and $N_{\theta}^{f}$ throughout the paper.

Fridy and Orhan [12] introduced the concept of lacunary statistical convergence as follows. 
Definition 5. Let $\theta=\left(k_{r}\right)$ be a lacunary sequence. A number sequence $x=\left(x_{k}\right)$ is said to be lacunary statistically convergent to $l$ or $S_{\theta}$-convergent to $l$, if, for each $\varepsilon>0$,

$$
\lim _{r \rightarrow \infty} \frac{1}{h_{r}}\left|\left\{k \in I_{r}:\left|x_{k}-l\right| \geq \varepsilon\right\}\right|=0 .
$$

In this case, one writes $S_{\theta}-\lim x_{k}=l$ or $x_{k} \rightarrow l\left(S_{\theta}\right)$. The set of all lacunary statistically convergent sequences is denoted by $S_{\theta}$.

Throughout this paper $s, l_{\infty}$, and $c$ will denote the spaces of all, bounded, and convergent sequences of complex numbers, respectively. Moreover, we will be concerned only with the sequences of scalars.

Following a very recent and new approach of Aizpuru et al. [5], we first introduced the notion of $f_{\alpha}$-density and, consequently, obtained a new concept of nonmatrix convergence, namely, $f$-statistical convergence of order $\alpha$ [6], where $f$ is an unbounded modulus function and $\alpha \in(0,1]$. The $f_{\alpha}$-density of the subset $A$ of $\mathbb{N}$ is defined as follows.

Definition 6. Let $f$ be an unbounded modulus function and let $\alpha$ be any real number such that $0<\alpha \leq 1$. Then

$$
d_{\alpha}^{f}(A)=\lim _{n \rightarrow \infty} \frac{f(|k \leq n: k \in A|)}{f\left(n^{\alpha}\right)}
$$

in case this limit exists, where $|k \leq n: k \in A|$ denotes the number of elements of $A$ not exceeding $n$.

Now, in this paper we introduce a new concept of $f$ lacunary statistical convergence, where $f$ is an unbounded modulus, as follows.

Definition 7. Let $f$ be an unbounded modulus and let $\theta=\left(k_{r}\right)$ be a lacunary sequence. A sequence $x=\left(x_{k}\right)$ is said to be $f$ lacunary statistically convergent to $l$ or $S_{\theta}^{f}$-convergent to $l$, if, for each $\varepsilon>0$,

$$
\lim _{r \rightarrow \infty} \frac{1}{f\left(h_{r}\right)} f\left(\left|\left\{k \in I_{r}:\left|x_{k}-l\right| \geq \varepsilon\right\}\right|\right)=0 .
$$

In this case, one writes $S_{\theta}^{f}-\lim x_{k}=l$ or $x_{k} \rightarrow l\left(S_{\theta}^{f}\right)$.

For a given lacunary sequence $\theta=\left(k_{r}\right)$ and an unbounded modulus $f$, by $S_{\theta}^{f}$ we denote the set of all $f$ lacunary statistically convergent sequences.

Remark 8. In case $f(x)=x$, the concept of $f$-lacunary statistical convergence reduces to that of lacunary statistical convergence.

In the next section it is shown that the concept of $f$ lacunary statistical convergence is intermediate between the ordinary convergence and the lacunary statistical convergence. It is also proved that, under certain conditions on a modulus $f$, if a sequence is lacunary strongly convergent with respect to a modulus $f$ then it is $f$-lacunary statistically convergent and that the concepts of lacunary strong convergence with respect to a modulus $f$ and $f$-lacunary statistical convergence are equivalent on bounded sequences. In Section 3, we characterize those $\theta$ for which $S^{f}=S_{\theta}^{f}$, where $f$ is an unbounded modulus such that $\lim _{t \rightarrow \infty}(f(t) / t)>$ 0 and there is a positive constant $c$ such that $f(x y) \geq$ $c f(x) f(y)$, for all $x \geq 0, y \geq 0$. Note that it was shown by Maddox [18] that, for any modulus $f, \lim _{t \rightarrow \infty}(f(t) / t)$ exists. Maddox [28] also showed that there exists an unbounded modulus $f$ for which there is a positive constant $c$ such that $f(x y) \geq c f(x) f(y)$, for all $x \geq 0, y \geq 0$. In Section 4 , we first observe that it is possible for a sequence to have different $S_{\theta}^{f}$-limits for different $f$ and $\theta$. In Theorem 24, we investigate certain conditions under which this situation cannot occur. In Section 5, a general description of inclusion between two arbitrary lacunary methods of $f$-statistical convergence is given. In the last section, $f$-lacunary statistical analog of Cauchy criterion for convergence is established. A Tauberian theorem for $S_{\theta}^{f}$-convergence is also given.

\section{2. $f$-Lacunary Statistical Convergence}

We begin by establishing elementary connections between convergence, $f$-lacunary statistical convergence, and lacunary statistical convergence.

Theorem 9. Every convergent sequence is f-lacunary statistically convergent; that is, $c \subset S_{\theta}^{f}$ for any unbounded modulus $f$ and lacunary sequence $\theta$.

Proof. Let $x=\left(x_{k}\right)$ be any convergent sequence. Then, for each $\epsilon>0$, the set $\left\{k \in \mathbb{N}:\left|x_{k}-l\right| \geq \epsilon\right\}$ is finite. Suppose $\left|\left\{k \in \mathbb{N}:\left|x_{k}-l\right| \geq \epsilon\right\}\right|=m_{o}$. Now, since $\left\{k \in I_{r}:\left|x_{k}-l\right| \geq\right.$ $\epsilon\} \subset\left\{k \in \mathbb{N}:\left|x_{k}-l\right| \geq \epsilon\right\}$ and $f$ being modulus is increasing, therefore

$$
\frac{f\left(\left|\left\{k \in I_{r}:\left|x_{k}-l\right| \geq \epsilon\right\}\right|\right)}{f\left(h_{r}\right)} \leq \frac{f\left(m_{o}\right)}{f\left(h_{r}\right)} .
$$

Taking limit as $r \rightarrow \infty$, on both sides, we get

$$
\begin{aligned}
\lim _{r \rightarrow \infty} \frac{f\left(\left|\left\{k \in I_{r}:\left|x_{k}-l\right| \geq \epsilon\right\}\right|\right)}{f\left(h_{r}\right)} & =0, \\
\text { as } f\left(h_{r}\right) & \longrightarrow \infty \text { as } r \longrightarrow \infty .
\end{aligned}
$$

Remark 10. The inclusion $c \subset S_{\theta}^{f}$ is strict as the sequence $x=$ $\left(x_{k}\right)$ given in the necessity part of Lemma 19 is an example of a sequence which is $S_{\theta}^{f}$-convergent but not convergent.

Theorem 11. Every $f$-lacunary statistically convergent sequence is lacunary statistically convergent.

Proof. Suppose $x=\left(x_{k}\right)$ is $f$-lacunary statistically convergent to $l$. Then by the definition of limit and the fact that $f$ 
being modulus is subadditive, for every $p \in \mathbb{N}$, there exists $r_{o} \in \mathbb{N}$ such that, for $r \geq r_{o}$, we have

$$
\begin{aligned}
& f\left(\left|\left\{k \in I_{r}:\left|x_{k}-l\right| \geq \epsilon\right\}\right|\right) \leq \frac{1}{p} f\left(h_{r}\right) \leq \frac{1}{p} p f\left(\frac{h_{r}}{p}\right) \\
& =f\left(\frac{h_{r}}{p}\right)
\end{aligned}
$$

and since $f$ is increasing, we have

$$
\frac{1}{h_{r}}\left|\left\{k \in I_{r}:\left|x_{k}-l\right| \geq \epsilon\right\}\right| \leq \frac{1}{p} .
$$

Hence, $x$ is lacunary statistically convergent to $l$.

Remark 12. It seems that the inclusion $S_{\theta}^{f} \subset S_{\theta}$ is strict. But right now we are not in a position to give an example of a sequence which is $S_{\theta}$-convergent but not $S_{\theta}^{f}$-convergent. So it is left as an open problem.

Remark 13. From Theorems 9 and 11, we can say that the concept of $f$-lacunary statistical convergence is intermediate between the usual notion of convergence and the lacunary statistical convergence.

We now establish a relationship between $f$-lacunary statistical convergence and lacunary strong convergence with respect to a modulus $f$. Fridy and Orhan [12] showed that on bounded sequences the concept of lacunary statistical convergence is equivalent to lacunary strong convergence. We now wish to find some condition on $f$, if any, so that the concept of $f$-lacunary statistical convergence becomes equivalent to lacunary strong convergence with respect to a modulus $f$.

Theorem 14. Let $\theta=\left(k_{r}\right)$ be a lacunary sequence; then consider the following:

(a) For any unbounded modulus $f$ for which $\lim _{t \rightarrow \infty}(f(t) / t)>0$ and there is a positive constant $c$ such that $f(x y) \geq c f(x) f(y)$, for all $x \geq 0, y \geq 0$,

$$
\text { (i) } x_{k} \rightarrow l\left(N_{\theta}^{f}\right) \text { implies } x_{k} \rightarrow l\left(S_{\theta}^{f}\right) \text {, }
$$

(ii) $N_{\theta}^{f}$ is a proper subset of $S_{\theta}^{f}$.

(b) $x \in l_{\infty}$ and $x_{k} \rightarrow l\left(S_{\theta}^{f}\right)$ imply $x_{k} \rightarrow l\left(N_{\theta}^{f}\right)$, for any unbounded modulus $f$.

(c) $N_{\theta}^{f} \cap l_{\infty}=S_{\theta}^{f} \cap l_{\infty}$ for any unbounded modulus $f$ for which $\lim _{t \rightarrow \infty}(f(t) / t)>0$ and there is a positive constant $c$ such that $f(x y) \geq c f(x) f(y)$ for all $x \geq 0$, $y \geq 0$.

Proof. (a) (i) For any sequence $x=\left(x_{k}\right)$ and $\epsilon>0$, by the definition of a modulus function (ii) and (iii) we have

$$
\frac{1}{h_{r}} \sum_{k \in I_{r}} f\left(\left|x_{k}-l\right|\right) \geq \frac{1}{h_{r}} f\left(\sum_{k \in I_{r}}\left|x_{k}-l\right|\right)
$$

$$
\begin{aligned}
& \geq \frac{1}{h_{r}} f\left(\sum_{\substack{k \in I_{r} \\
\left|x_{k}-l\right| \geq \epsilon}}\left|x_{k}-l\right|\right) \\
& \geq \frac{1}{h_{r}} f\left(\left|\left\{k \in I_{r}:\left|x_{k}-l\right| \geq \varepsilon\right\}\right| \epsilon\right) \\
& \geq \frac{c}{h_{r}} f\left(\left|\left\{k \in I_{r}:\left|x_{k}-l\right| \geq \varepsilon\right\}\right|\right) f(\epsilon) \\
& =\frac{c}{h_{r}} \frac{f\left(\left|\left\{k \in I_{r}:\left|x_{k}-l\right| \geq \varepsilon\right\}\right|\right)}{f\left(h_{r}\right)} f\left(h_{r}\right) f(\epsilon)
\end{aligned}
$$

from where it follows that $x \in S_{\theta}^{f}$ as $x \in N_{\theta}^{f}$ and $\lim _{r \rightarrow \infty}\left(f\left(h_{r}\right) / h_{r}\right)>0$.

(ii) To show the strictness of inclusion, consider the sequence $x=\left(x_{k}\right)$ such that $x_{k}$ is to be $1,2, \ldots,\left[\sqrt{h_{r}}\right]$ at the first $\left[\sqrt{h_{r}}\right]$ integers in $I_{r}$, and $x_{k}=0$ otherwise. Note that $\left(x_{k}\right)$ is not bounded. Also, for every $\epsilon>0$,

$$
\begin{gathered}
\frac{1}{f\left(h_{r}\right)} f\left(\left|\left\{k \in I_{r}:\left|x_{k}-0\right| \geq \varepsilon\right\}\right|\right)=\frac{f\left(\left[\sqrt{h_{r}}\right]\right)}{f\left(h_{r}\right)} \\
=\frac{f\left(\left[\sqrt{h_{r}}\right]\right)}{\left[\sqrt{h_{r}}\right]} \times \frac{h_{r}}{f\left(h_{r}\right)} \times \frac{\left[\sqrt{h_{r}}\right]}{h_{r}} \longrightarrow 0
\end{gathered}
$$

as $r \longrightarrow \infty$,

because $\lim _{r \rightarrow \infty}\left(f\left(\left[\sqrt{h_{r}}\right]\right) /\left[\sqrt{h_{r}}\right]\right), \lim _{r \rightarrow \infty}\left(f\left(h_{r}\right) / h_{r}\right) \quad$ are positive and $\lim _{r \rightarrow \infty}\left(\left[\sqrt{h_{r}}\right] / h_{r}\right)=0$.

Thus, $x_{k} \rightarrow 0\left(S_{\theta}^{f}\right)$. On the other hand,

$$
\begin{aligned}
& \frac{1}{h_{r}} \sum_{k \in I_{r}} f\left(\left|x_{k}-0\right|\right)=\frac{f(1)+f(2)+\cdots+f\left(\left[\sqrt{h_{r}}\right]\right)}{h_{r}} \\
& \geq \frac{f\left(1+2+\cdots+\left[\sqrt{h_{r}}\right]\right)}{h_{r}} \\
& =\frac{f\left(\left[\sqrt{h_{r}}\right]\left(\left[\sqrt{h_{r}}\right]+1\right) / 2\right)}{h_{r}} \\
& \geq c \frac{f\left(\left[\sqrt{h_{r}}\right]\right) f\left(\left(\left[\sqrt{h_{r}}\right]+1\right) / 2\right)}{h_{r}} \\
& =c \times \frac{f\left(\left[\sqrt{h_{r}}\right]\right)}{\left[\sqrt{h_{r}}\right]}
\end{aligned}
$$




$$
\begin{aligned}
& \times \frac{f\left(\left(\left[\sqrt{h_{r}}\right]+1\right) / 2\right)}{\left(\left[\sqrt{h_{r}}\right]+1\right) / 2} \\
& \times \frac{\left[\sqrt{h_{r}}\right]\left(\left(\left[\sqrt{h_{r}}\right]+1\right) / 2\right)}{h_{r}}>0
\end{aligned}
$$

as $c, \lim _{r \rightarrow \infty}\left(f\left(\left[\sqrt{h_{r}}\right]\right) /\left[\sqrt{h_{r}}\right]\right), \lim _{r \rightarrow \infty}\left(f\left(\left(\left[\sqrt{h_{r}}\right]+1\right) / 2\right) /\right.$ $\left.\left(\left[\sqrt{h_{r}}\right]+1\right) / 2\right)$, and $\lim _{r \rightarrow \infty}\left(\left[\sqrt{h_{r}}\right]\left(\left(\left[\sqrt{h_{r}}\right]+1\right) / 2\right) / h_{r}\right)$ are positive. Hence $x_{k} \nrightarrow 0\left(N_{\theta}^{f}\right)$.

(b) Suppose that $x_{k} \rightarrow l\left(S_{\theta}^{f}\right)$ and $x \in l_{\infty}$; say $\left|x_{k}-l\right| \leq H$ for all $k \in \mathbb{N}$. Given $\epsilon>0$, we have

$$
\begin{aligned}
\frac{1}{h_{r}} \sum_{k \in I_{r}} f\left(\left|x_{k}-l\right|\right)= & \frac{1}{h_{r}} \sum_{\substack{k \in I_{r} \\
\left|x_{k}-l\right| \geq \epsilon}} f\left(\left|x_{k}-l\right|\right) \\
& +\frac{1}{h_{r}} \sum_{\substack{k \in I_{r} \\
\left|x_{k}-l\right|<\epsilon}} f\left(\left|x_{k}-l\right|\right) \\
\leq & \frac{1}{h_{r}}\left|\left\{k \in I_{r}:\left|x_{k}-l\right| \geq \varepsilon\right\}\right| f(H) \\
& +\frac{1}{h_{r}} h_{r} f(\epsilon) .
\end{aligned}
$$

Taking limit on both sides as $r \rightarrow \infty$, we get $\lim _{r \rightarrow \infty}\left(1 / h_{r}\right) \sum_{k \in I_{r}} f\left(\left|x_{k}-l\right|\right)=0$, in view of Theorem 11 and the fact that $f$ is increasing.

(c) This is an immediate consequence of (a) and (b).

Remark 15. The example given in part (a) of the above theorem shows that the boundedness condition cannot be omitted from the hypothesis of part (b).

Remark 16. If we take $f(x)=x$ in Theorem 14, we obtain Theorem 1 of Fridy and Orhan [12].

\section{3. $f$-Lacunary Statistical Convergence versus $f$-Statistical Convergence}

In this section we study the inclusions $S_{\theta}^{f} \subset S^{f}$ and $S^{f} \subset S_{\theta}^{f}$ under certain restrictions on $\theta$ and $f$.

Lemma 17. For any lacunary sequence $\theta$ and unbounded modulus $f$ for which $\lim _{t \rightarrow \infty}(f(t) / t)>0$ and there is $a$ positive constant $c$ such that $f(x y) \geq c f(x) f(y)$, for all $x \geq 0$, $y \geq 0$, one has $S^{f} \subset S_{\theta}^{f}$ if and only if $\liminf _{r} q_{r}>1$.

\section{Proof.}

Sufficiency. If $\lim \inf _{r} q_{r}>1$, then there exists $\delta>0$ such that $q_{r} \geq 1+\delta$ for sufficiently large $r$. Since $h_{r}=k_{r}-k_{r-1}$, we have

$$
\frac{h_{r}}{k_{r}} \geq \frac{\delta}{1+\delta}
$$

for sufficiently large $r$. If $x_{k} \rightarrow l\left(S^{f}\right)$, then, for given $\epsilon>0$ and sufficiently large $r$, we have

$$
\begin{aligned}
& \frac{1}{f\left(k_{r}\right)} f\left(\left|\left\{k \leq k_{r}:\left|x_{k}-l\right| \geq \varepsilon\right\}\right|\right) \\
& \geq \frac{f\left(\left|\left\{k \in I_{r}:\left|x_{k}-l\right| \geq \varepsilon\right\}\right|\right)}{f\left(k_{r}\right)}=\frac{f\left(h_{r}\right)}{f\left(k_{r}\right)} \\
& \quad \times \frac{f\left(\left|\left\{k \in I_{r}:\left|x_{k}-l\right| \geq \varepsilon\right\}\right|\right)}{f\left(h_{r}\right)}=\left(\frac{f\left(h_{r}\right)}{h_{r}}\right) \\
& \cdot\left(\frac{k_{r}}{f\left(k_{r}\right)}\right)\left(\frac{h_{r}}{k_{r}}\right) \frac{f\left(\left|\left\{k \in I_{r}:\left|x_{k}-l\right| \geq \varepsilon\right\}\right|\right)}{f\left(h_{r}\right)} \\
& \geq\left(\frac{f\left(h_{r}\right)}{h_{r}}\right)\left(\frac{k_{r}}{f\left(k_{r}\right)}\right)\left(\frac{\delta}{1+\delta}\right) \\
& . \frac{f\left(\left|\left\{k \in I_{r}:\left|x_{k}-l\right| \geq \varepsilon\right\}\right|\right)}{f\left(h_{r}\right)} .
\end{aligned}
$$

This proves the sufficiency.

Necessity. Assume that $\liminf _{r} q_{r}=1$. Proceeding as in Lemma 2.1 of [27], we can select a subsequence $\left(k_{r(j)}\right)$ of $\theta$ satisfying

$$
\begin{aligned}
& \frac{k_{r(j)}}{k_{r(j)-1}}<1+\frac{1}{j}, \\
& \frac{k_{r(j)-1}}{k_{r(j-1)}}>j,
\end{aligned}
$$

where $r(j) \geq r(j-1)+2$.

Define a bounded sequence $x=\left(x_{k}\right)$ by

$$
x_{k}= \begin{cases}1 & \text { if } k \in I_{r(j)}, \text { for some } j=1,2,3, \ldots \\ 0 & \text { otherwise. }\end{cases}
$$

It is shown in Lemma 2.1 of [27] that $x \notin N_{\theta}$ but $x \in w$. Thus in view of Theorem 3 of [29] and Theorem 14 we have $x \notin S_{\theta}^{f}$. On the other hand, it follows from Theorem 4 of [28] and Corollary 4.3 of [6] that $x \in S^{f}$. Hence $S^{f} \not \subset S_{\theta}^{f}$. But this is a contradiction to the assumption that $S^{f} \subset S_{\theta}^{f}$. This contradiction shows that our assumption is wrong. Hence $\liminf _{r} q_{r}>1$.

Remark 18. The sequence $x=\left(x_{k}\right)$, constructed in the necessity part of the above lemma, is an example of $f$ statistically convergent sequence which is not $f$-lacunary statistically convergent.

Lemma 19. For any lacunary sequence $\theta$ and unbounded modulus $f$ for which $\lim _{t \rightarrow \infty}(f(t) / t)>0$ and there is $a$ positive constant $c$ such that $f(x y) \geq c f(x) f(y)$, for all $x \geq$ $0, y \geq 0$, one has $S_{\theta}^{f} \subset S^{f}$ if and only if $\lim \sup _{r} q_{r}<\infty$. 
Proof.

Sufficiency. If $\limsup _{r} q_{r}<\infty$, then there is $H>0$ such that $q_{r}<H$ for all $r$. Now, suppose that $x_{k} \rightarrow l\left(S_{\theta}^{f}\right)$ and $\lim _{r \rightarrow \infty}\left(f\left(h_{r}\right) / h_{r}\right)=l^{\prime}$. Therefore, for given $\epsilon>0$, there exists $r_{o} \in \mathbb{N}$ such that for all $r>r_{o}$

$$
\begin{array}{r}
\frac{f\left(h_{r}\right)}{h_{r}}<l^{\prime}+\epsilon, \\
\frac{1}{f\left(h_{r}\right)} f\left(\left|\left\{k \in I_{r}:\left|x_{k}-l\right| \geq \varepsilon\right\}\right|\right)<\epsilon .
\end{array}
$$

Let $N_{r}=\left|\left\{k \in I_{r}:\left|x_{k}-l\right| \geq \epsilon\right\}\right|$. Using this notation, we have

$$
\frac{f\left(N_{r}\right)}{f\left(h_{r}\right)}<\epsilon \quad \forall r>r_{o} .
$$

Now, let $M=\max \left\{f\left(N_{1}\right), f\left(N_{2}\right), \ldots f\left(N_{r_{o}}\right)\right\}$ and let $n$ be an integer such that $k_{r-1}<n \leq k_{r}$, and then we can write

$$
\begin{aligned}
& \frac{1}{f(n)} f\left(\left|\left\{k \leq n:\left|x_{k}-l\right| \geq \varepsilon\right\}\right|\right) \leq \frac{1}{f\left(k_{r-1}\right)} \\
& \cdot f\left(\left|\left\{k \leq k_{r}:\left|x_{k}-l\right| \geq \varepsilon\right\}\right|\right)=\frac{1}{f\left(k_{r-1}\right)} f\left(N_{1}\right. \\
& \left.+N_{2}+\cdots+N_{r_{o}}+N_{r_{o}+1}+\cdots+N_{r}\right) \\
& \leq \frac{1}{f\left(k_{r-1}\right)}\left(f\left(N_{1}\right)+f\left(N_{2}\right)+\cdots+f\left(N_{r_{o}}\right)\right. \\
& \left.+f\left(N_{r_{o}+1}\right)+\cdots+f\left(N_{r}\right)\right) \leq \frac{r_{o} M}{f\left(k_{r-1}\right)} \\
& +\frac{1}{f\left(k_{r-1}\right)}\left[f\left(N_{r_{o}+1}\right)+\cdots+f\left(N_{r}\right)\right]=\frac{r_{o} M}{f\left(k_{r-1}\right)} \\
& +\frac{1}{f\left(k_{r-1}\right)}\left[\frac{f\left(h_{r_{o}+1}\right)}{h_{r_{o}+1}} \frac{f\left(N_{r_{o}+1}\right)}{f\left(h_{r_{o}+1}\right)} h_{r_{o}+1}+\cdots\right. \\
& \left.+\frac{f\left(h_{r}\right)}{h_{r}} \frac{f\left(N_{r}\right)}{f\left(h_{r}\right)} h_{r}\right]<\frac{r_{o} M}{f\left(k_{r-1}\right)} \\
& +\frac{1}{f\left(k_{r-1}\right)}\left[\left(l^{\prime}+\epsilon\right) \epsilon h_{r_{o}+1}+\cdots+\left(l^{\prime}+\epsilon\right) \epsilon h_{r}\right] \\
& =\frac{r_{o} M}{f\left(k_{r-1}\right)}+\frac{1}{f\left(k_{r-1}\right)} \epsilon\left(l^{\prime}+\epsilon\right)\left[h_{r_{o}+1}+\cdots+h_{r}\right] \\
& =\frac{r_{o} M}{f\left(k_{r-1}\right)}+\frac{1}{f\left(k_{r-1}\right)} \epsilon\left(l^{\prime}+\epsilon\right)\left[k_{r}-k_{r_{o}}\right] \\
& <\frac{r_{o} M}{f\left(k_{r-1}\right)}+\epsilon\left(l^{\prime}+\epsilon\right)\left[\frac{k_{r}}{f\left(k_{r-1}\right)}\right]=\frac{r_{o} M}{f\left(k_{r-1}\right)}
\end{aligned}
$$

$$
\begin{aligned}
& +\epsilon\left(l^{\prime}+\epsilon\right) \frac{1}{f\left(k_{r-1}\right) / k_{r-1}} \frac{k_{r}}{k_{r-1}}=\frac{r_{o} M}{f\left(k_{r-1}\right)}+\epsilon\left(l^{\prime}\right. \\
& +\epsilon) q_{r} \frac{1}{f\left(k_{r-1}\right) / k_{r-1}}<\frac{r_{o} M}{f\left(k_{r-1}\right)}+\epsilon\left(l^{\prime}+\epsilon\right) H \\
& \cdot \frac{1}{f\left(k_{r-1}\right) / k_{r-1}},
\end{aligned}
$$

from where the sufficiency follows immediately, in view of the fact that $\lim _{r \rightarrow \infty}\left(f\left(k_{r-1}\right) / k_{r-1}\right)>0$.

Necessity. Suppose that $\lim \sup _{r} q_{r}=\infty$. Following Lemma 2.2 of [27], we can select a subsequence $\left(k_{r(j)}\right)$ of lacunary sequence $\theta$ such that $q_{r(j)}>j$. Define a bounded sequence $x=\left(x_{k}\right)$ by

$$
\begin{aligned}
& x_{k} \\
& = \begin{cases}1 & \text { if } k_{r(j)-1}<k \leq 2 k_{r(j)-1}, \text { for some } j=1,2,3, \ldots ; \\
0 & \text { otherwise. }\end{cases}
\end{aligned}
$$

It is shown in [27], on page 511, that $x \in N_{\theta}$ but $x \notin w$. By Theorem 3 of [29] and Theorem 14, we conclude that $x \in S_{\theta}^{f}$, but $x \notin S^{f}$, in view of Theorem 2.1 of [10] and the fact that every $f$-statistically convergent sequence is statistically convergent. Hence $S_{\theta}^{f} \not \subset S^{f}$. But this is a contradiction to the assumption that $S_{\theta}^{f} \subset S^{f}$. This contradiction shows that $\lim \sup _{r} q_{r}<\infty$.

Remark 20. The sequence $x=\left(x_{k}\right)$, constructed in the necessity part of the above lemma, is an example of $f$ lacunary statistically convergent sequence which is not $f$ statistically convergent.

Combining Lemmas 17 and 19 we have the following.

Theorem 21. For any lacunary sequence $\theta$ and unbounded modulus $f$ for which $\lim _{t \rightarrow \infty}(f(t) / t)>0$ and there is a positive constant $c$ such that $f(x y) \geq c f(x) f(y)$, for all $x \geq 0$, $y \geq 0$, one has $S_{\theta}^{f}=S^{f}$ if and only if $1<\liminf _{r} q_{r} \leq$ $\lim \sup _{r} q_{r}<\infty$.

Theorem 22. For any lacunary sequence $\theta$ and unbounded modulus $f$ for which $\lim _{t \rightarrow \infty}(f(t) / t)>0$ and there is a positive constant $c$ such that $f(x y) \geq c f(x) f(y)$, for all $x \geq 0$, $y \geq 0$, one has

$$
S^{f}=\bigcap_{\lim \inf _{r} q_{r}>1} S_{\theta}^{f}=\bigcup_{\lim \sup _{r} q_{r}<\infty} S_{\theta}^{f} .
$$

Proof. In view of Lemma 17, we have $S^{f} \subset \bigcap_{\lim \inf _{r} q_{r}>1} S_{\theta}^{f}$. Suppose if possible $x=\left(x_{k}\right) \in \bigcap_{\lim _{\inf _{r}} q_{r}>1} S_{\theta}^{f}$ but $x \notin S^{f}$. We have $\left(x_{k}\right) \in S_{\theta}^{f}$ for all $\theta=\left(k_{r}\right)$ for which $\liminf { }_{r} q_{r}>1$. If we take $\theta=\left(2^{r}\right)$, then, in view of Theorem 21, we have $S_{\theta}^{f}=$ $S^{f}$ and so $x \in S^{f}$, contrary to our assumption. Hence $S^{f}=$ $\bigcap_{\text {lim } \inf _{r} q_{r}>1} S_{\theta}^{f}$. The remaining part can be proved similarly and hence is omitted. 
Remark 23. The sequence $x=\left(x_{k}\right)$ constructed in part (a) of Theorem 14 belongs to $S_{\theta}^{f}$ for every lacunary sequence $\theta$, as well as unbounded modulus $f$ for which $\lim _{t \rightarrow \infty}(f(t) / t)>0$ and there is a positive constant $c$ such that $f(x y) \geq c f(x) f(y)$ for all $x \geq 0, y \geq 0$. Hence $\bigcap_{\lim \inf _{r} q_{r}>1} S_{\theta}^{f} \neq \phi$.

\section{Uniqueness of $S_{\theta}^{f}$-Limit}

For any fixed $\theta$, the $S_{\theta}$-limit is unique; however, Fridy and Orhan [12] showed that it is possible for a sequence to have different $S_{\theta}$-limits for different $\theta$ 's. They showed that this situation cannot occur if the sequence is statistically convergent. We now establish a similar result in case of $S_{\theta}^{f}$ convergence. First we observe that it is possible for a sequence to have different $S_{\theta}^{f}$-limits for different $f$ and $\theta$. To illustrate this, let us take $f(x)=x, g(x)=2 x, \theta_{1}=((2 r) !)$, and $\theta_{2}=((2 r+1) !)$. Consider the sequence $x$ given in [27], in the proof of Theorem 2.1, for which $N_{\theta_{1}}-\lim x_{k}=0$ and $N_{\theta_{2}}-\lim x_{k}=1$. Now, by applying Theorem 3 of [29] and Theorem 14 part (a), we see that $S_{\theta_{1}}^{f}-\lim x_{k}=0$ and $S_{\theta_{2}}^{g}-\lim x_{k}=1$.

In the next theorem we investigate certain conditions under which this situation cannot occur.

Theorem 24. For any two lacunary sequences $\theta_{1}$ and $\theta_{2}$, if $x \in$ $S^{f} \cap S_{\theta_{1}}^{f}$ and $S^{g} \cap S_{\theta_{2}}^{g}$, then $S_{\theta_{1}}^{f}-\lim x_{k}=S_{\theta_{2}}^{g}-\lim x_{k}$, where $f$ and $g$ are unbounded modulus functions for which

$$
\begin{gathered}
|f(x)-f(y)|=f(|x-y|), \\
|g(x)-g(y)|=g(|x-y|),
\end{gathered}
$$

$$
\forall x \geq 0, \quad y \geq 0 .
$$

To prove this theorem we need the following lemma.

Lemma 25. For any lacunary sequence $\theta$, if $x \in S^{f} \cap S_{\theta}^{f}$, then $S_{\theta}^{f}-\lim x_{k}=S^{f}-\lim x_{k}$, where $f$ is an unbounded modulus function for which

$$
|f(x)-f(y)|=f(|x-y|), \quad \forall x \geq 0, \quad y \geq 0 .
$$

Proof. Suppose $S^{f}-\lim x_{k}=l$ and $S_{\theta}^{f}-\lim x_{k}=l^{\prime}$, and $l \neq l^{\prime}$. Let $\epsilon>0$ be such that $0<\epsilon<\left|l-l^{\prime}\right| / 2$. Using the definition of a modulus (iii) and (ii), we have

$$
\begin{aligned}
& \frac{f\left(\left|\left\{k \leq n:\left|l-l^{\prime}\right| \geq 2 \varepsilon\right\}\right|\right)}{f(n)} \\
& \leq \frac{f\left(\left|\left\{k \leq n:\left|x_{k}-l\right| \geq \varepsilon\right\}\right|\right)}{f(n)} \\
& +\frac{f\left(\left|\left\{k \leq n:\left|x_{k}-l^{\prime}\right| \geq \varepsilon\right\}\right|\right)}{f(n)} .
\end{aligned}
$$

Taking limit as $n \rightarrow \infty$ on both sides, we get

$$
\begin{aligned}
& 1 \leq 0+\lim _{n \rightarrow \infty} \frac{f\left(\left|\left\{k \leq n:\left|x_{k}-l^{\prime}\right| \geq \varepsilon\right\}\right|\right)}{f(n)} \leq 1, \\
& \text { hence } \lim _{n \rightarrow \infty} \frac{f\left(\left|\left\{k \leq n:\left|x_{k}-l^{\prime}\right| \geq \varepsilon\right\}\right|\right)}{f(n)}=1 .
\end{aligned}
$$

Now consider the $k_{m}$ th term of the sequence $\left((f(n))^{-1} f(\mid\{k \leq\right.$ $\left.\left.\left.n:\left|x_{k}-l^{\prime}\right| \geq \varepsilon\right\} \mid\right)\right)$ :

$$
\begin{gathered}
\frac{1}{f\left(k_{m}\right)} f\left(\left|\left\{k \leq k_{m}:\left|x_{k}-l^{\prime}\right| \geq \varepsilon\right\}\right|\right)=\frac{1}{f\left(k_{m}\right)} \\
\cdot f\left(\left|\left\{k \in \bigcup_{r=1}^{m} I_{r}:\left|x_{k}-l^{\prime}\right| \geq \varepsilon\right\}\right|\right)=\frac{1}{f\left(k_{m}\right)} \\
\cdot f\left(\sum_{r=1}^{m}\left|\left\{k \in I_{r}:\left|x_{k}-l^{\prime}\right| \geq \varepsilon\right\}\right|\right) \leq \frac{1}{f\left(k_{m}\right)} \\
\cdot \sum_{r=1}^{m} f\left(\left|\left\{k \in I_{r}:\left|x_{k}-l^{\prime}\right| \geq \varepsilon\right\}\right|\right)=\frac{1}{f\left(k_{m}\right)} \\
\cdot \sum_{r=1}^{m} f\left(h_{r}\right) \frac{1}{f\left(h_{r}\right)} f\left(\left|\left\{k \in I_{r}:\left|x_{k}-l^{\prime}\right| \geq \varepsilon\right\}\right|\right) .
\end{gathered}
$$

Also, in view of the choice of unbounded modulus $f$, we have

$$
\begin{aligned}
\sum_{r=1}^{m} f\left(h_{r}\right)= & f\left(h_{1}\right)+f\left(h_{2}\right)+\cdots+f\left(h_{m}\right) \\
= & f\left(k_{1}-k_{o}\right)+f\left(k_{2}-k_{1}\right)+\cdots \\
& +f\left(k_{m}-k_{m-1}\right) \\
= & f\left(\left|k_{1}-k_{o}\right|\right)+f\left(\left|k_{2}-k_{1}\right|\right)+\cdots \\
& +f\left(\left|k_{m}-k_{m-1}\right|\right) \\
= & \left|f\left(k_{1}\right)-f\left(k_{o}\right)\right|+\left|f\left(k_{2}\right)-f\left(k_{1}\right)\right|+\cdots \\
& +\left|f\left(k_{m}\right)-f\left(k_{m-1}\right)\right| \\
= & f\left(k_{1}\right)-f\left(k_{o}\right)+f\left(k_{2}\right)-f\left(k_{1}\right)+\cdots \\
& +f\left(k_{m}\right)-f\left(k_{m-1}\right)=f\left(k_{m}\right) .
\end{aligned}
$$

Now, using (33) in (32), we have

$$
\begin{aligned}
& \frac{1}{f\left(k_{m}\right)} f\left(\left|\left\{k \leq k_{m}:\left|x_{k}-l^{\prime}\right| \geq \varepsilon\right\}\right|\right) \\
& \leq \frac{1}{\sum_{r=1}^{m} f\left(h_{r}\right)} \sum_{r=1}^{m} f\left(h_{r}\right) t_{r},
\end{aligned}
$$

where $t_{r}=\left(f\left(h_{r}\right)\right)^{-1} f\left(\left|\left\{k \in I_{r}:\left|x_{k}-l^{\prime}\right| \geq \varepsilon\right\}\right|\right) \rightarrow 0$ because $x_{k} \rightarrow l^{\prime}\left(S_{\theta}^{f}\right)$. Since $\theta$ is a lacunary sequence and $f$ being modulus is increasing, the term on the right hand side of (34) 
is a regular weighted mean transformation of $t=\left(t_{r}\right)$, and therefore it, too, tends to zero as $r \rightarrow \infty$. Thus

$$
\frac{1}{f\left(k_{m}\right)} f\left(\left|\left\{k \leq k_{m}:\left|x_{k}-l^{\prime}\right| \geq \varepsilon\right\}\right|\right) \longrightarrow 0
$$

$$
\text { as } m \longrightarrow \infty \text {. }
$$

Also, since $\left(f\left(k_{m}\right)\right)^{-1} f\left(\left|\left\{k \leq k_{m}:\left|x_{k}-l^{\prime}\right| \geq \varepsilon\right\}\right|\right)$ is a subsequence of sequence $(f(n))^{-1} f\left(\left|\left\{k \leq n:\left|x_{k}-l^{\prime}\right| \geq \varepsilon\right\}\right|\right)$, we conclude that

$$
(f(n))^{-1} f\left(\left|\left\{k \leq n:\left|x_{k}-l^{\prime}\right| \geq \varepsilon\right\}\right|\right) \nrightarrow 1 .
$$

But this is a contradiction to (31). This contradiction shows that $l=l^{\prime}$.

Proof of Theorem 24. By Lemma 25, we have

$$
\begin{aligned}
& S^{f}-\lim x_{k}=S_{\theta_{1}}^{f}-\lim x_{k}, \\
& S^{g}-\lim x_{k}=S_{\theta_{2}}^{g}-\lim x_{k} .
\end{aligned}
$$

But, according to Corollary 2.2 of Aizpuru et al. [5], we have

$$
S^{f}-\lim x_{k}=S^{g}-\lim x_{k}
$$

Therefore, from (37) and (38) we have

$$
S_{\theta_{1}}^{f}-\lim x_{k}=S_{\theta_{2}}^{g}-\lim x_{k}
$$

If we take $g=f$ in Theorem 24 we have the following.

Corollary 26. For any two lacunary sequences $\theta_{1}$ and $\theta_{2}$, if $x \in S^{f}$ and $x \in S_{\theta_{1}}^{f} \cap S_{\theta_{2}}^{f}$, then $S_{\theta_{1}}^{f}-\lim x_{k}=S_{\theta_{2}}^{f}-\lim x_{k}$, where $f$ is an unbounded modulus function such that

$$
|f(x)-f(y)|=f(|x-y|), \quad \forall x \geq 0, \quad y \geq 0 .
$$

If we take $f(x)=x$ in Corollary 26 we obtain the following result which contains Theorem 6 of Fridy and Orhan [12].

Corollary 27. For any two lacunary sequences $\theta_{1}$ and $\theta_{2}$, if $x \in S$ and $x \in S_{\theta_{1}} \cap S_{\theta_{2}}$, then $S_{\theta_{1}}-\lim x_{k}=S_{\theta_{2}} \lim x_{k}$.

If we take $\theta_{1}=\theta_{2}=\theta$ in Theorem 24 we have the following.

Corollary 28. For any lacunary sequence $\theta$, if $x \in S^{f} \cap S_{\theta}^{f}$ and $S^{g} \cap S_{\theta}^{g}$, then $S_{\theta}^{f}-\lim x_{k}=S_{\theta}^{g}-\lim x_{k}$, where $f$ and $g$ are unbounded modulus functions for which

$$
\begin{aligned}
|f(x)-f(y)| & =f(|x-y|), \\
|g(x)-g(y)| & =g(|x-y|), \quad \forall x \geq 0, \quad y \geq 0 .
\end{aligned}
$$

\section{Inclusion between Two Lacunary Methods of $f$-Statistical Convergence}

Our first result shows that, for certain $f$, if $\beta$ is a lacunary refinement of $\theta$, then $S_{\beta}^{f} \subset S_{\theta}^{f}$. To establish this result, we first recall the definition of a lacunary refinement of lacunary sequence [27].

Definition 29. The lacunary sequence $\beta^{\prime}=\left(k_{r}^{\prime}\right)$ is called a lacunary refinement of the lacunary sequence $\theta=\left(k_{r}\right)$ if $\left(k_{r}\right) \subset\left(k_{r}^{\prime}\right)$.

Theorem 30. If $\beta=\left(k_{r}^{\prime}\right)$ is a lacunary refinement of $\theta=\left(k_{r}\right)$ and $f$ is an unbounded modulus such that

$$
|f(x)-f(y)|=f(|x-y|), \quad \forall x \geq 0, \quad y \geq 0,
$$

then $x \in S_{\beta}^{f}$ implies $x \in S_{\theta}^{f}$.

Proof. Suppose each $I_{r}$ of $\theta$ contains the points $\left(k_{r, i}^{\prime}\right)_{i=1}^{v(r)}$ of $\beta$ so that

$$
\begin{aligned}
& k_{r-1}<k_{r, 1}^{\prime}<k_{r, 2}^{\prime}<\cdots<k_{r, v(r)}^{\prime}=k_{r} \\
& \quad \text { where } I_{r, i}^{\prime}=\left(k_{r, i-1}^{\prime}, k_{r, i}^{\prime}\right] .
\end{aligned}
$$

Note that, for all $r, v(r) \geq 1$ because $\left(k_{r}\right) \subset\left(k_{r}^{\prime}\right)$. Let $x_{k} \rightarrow$ $l\left(S_{\beta}^{f}\right)$. Therefore, for each $\epsilon>0$, we have

$$
\lim _{\substack{r \rightarrow \infty \\ 1 \leq i \leq \nu(r)}} \frac{1}{f\left(h_{r, i}^{\prime}\right)} f\left(\left|\left\{k \in I_{r, i}^{\prime}:\left|x_{k}-l\right| \geq \varepsilon\right\}\right|\right)=0,
$$

where $h_{r, i}^{\prime}=k_{r, i}^{\prime}-k_{r, i-1}^{\prime}$ and $h_{r, 1}^{\prime}=k_{r, 1}^{\prime}-k_{r-1}$, whence

$$
\lim _{r \rightarrow \infty} \sum_{\substack{I_{r, i}^{\prime} \subset I_{r} \\ 1 \leq i \leq \nu(r)}} \frac{1}{f\left(h_{r, i}^{\prime}\right)} f\left(\left|\left\{k \in I_{r, i}^{\prime}:\left|x_{k}-l\right| \geq \varepsilon\right\}\right|\right)=0 .
$$

For each $\epsilon>0$, we have

$$
\begin{aligned}
& \frac{1}{f\left(h_{r}\right)} f\left(\left|\left\{k \in I_{r}:\left|x_{k}-l\right| \geq \varepsilon\right\}\right|\right)=\frac{1}{f\left(h_{r}\right)} \\
& f\left(\left|\left\{k \in \bigcup_{\substack{I_{r, i}^{\prime} \subset I_{r} \\
1 \leq i \leq \nu(r)}} I_{r, i}^{\prime}:\left|x_{k}-l\right| \geq \varepsilon\right\}\right|\right) \\
& =\frac{1}{f\left(h_{r}\right)} f\left(\sum_{\substack{I_{r, i}^{\prime} I_{r} \\
1 \leq i \leq \nu(r)}}\left|\left\{k \in I_{r, i}^{\prime}:\left|x_{k}-l\right| \geq \varepsilon\right\}\right|\right)
\end{aligned}
$$




$$
\begin{aligned}
& \leq \frac{1}{f\left(h_{r}\right)} \sum_{\substack{I_{r, i}^{\prime} \subset I_{r} \\
1 \leq i \leq \nu(r)}} f\left(\left|\left\{k \in I_{r, i}^{\prime}:\left|x_{k}-l\right| \geq \varepsilon\right\}\right|\right) \\
& =\frac{1}{f\left(h_{r}\right)} \sum_{\substack{I_{r, i}^{\prime} \subset I_{r} \\
1 \leq i \leq \nu(r)}} f\left(h_{r, i}^{\prime}\right) \\
& \cdot \frac{1}{f\left(h_{r, i}^{\prime}\right)} f\left(\left|\left\{k \in I_{r, i}^{\prime}:\left|x_{k}-l\right| \geq \varepsilon\right\}\right|\right) .
\end{aligned}
$$

Also, in view of the choice of unbounded modulus $f$ and using the fact that $\beta=\left(k_{r}^{\prime}\right)$ is increasing, we have

$$
\begin{aligned}
\sum_{\substack{I_{r, i}^{\prime} \subset I_{r} \\
1 \leq i \leq \nu(r)}} f\left(h_{r, i}^{\prime}\right)= & f\left(h_{r, 1}^{\prime}\right)+f\left(h_{r, 2}^{\prime}\right)+\cdots+f\left(h_{r, v(r)}^{\prime}\right) \\
= & f\left(k_{r, 1}^{\prime}-k_{r-1}\right)+f\left(k_{r, 2}^{\prime}-k_{r, 1}^{\prime}\right) \\
& +\cdots+f\left(k_{r, v(r)}^{\prime}-k_{r, v(r)-1}^{\prime}\right) \\
= & f\left(\left|k_{r, 1}^{\prime}-k_{r-1}\right|\right)+f\left(\left|k_{r, 2}^{\prime}-k_{r, 1}^{\prime}\right|\right) \\
& +\cdots+f\left(\left|k_{r, \nu(r)}^{\prime}-k_{r, v(r)-1}^{\prime}\right|\right) \\
= & \left|f\left(k_{r, 1}^{\prime}\right)-f\left(k_{r-1}\right)\right| \\
& +\left|f\left(k_{r, 2}^{\prime}\right)-f\left(k_{r, 1}^{\prime}\right)\right|+\cdots \\
& +\left|f\left(k_{r, v(r)}^{\prime}\right)-f\left(k_{r, v(r)-1}^{\prime}\right)\right| \\
= & f\left(k_{r, 1}^{\prime}\right)-f\left(k_{r-1}\right)+f\left(k_{r, 2}^{\prime}\right) \\
& -f\left(k_{r, 1}^{\prime}\right)+\cdots+f\left(k_{r, v(r)}^{\prime}\right) \\
& -f\left(k_{r, v(r)-1}^{\prime}\right)=f\left(k_{r}\right)-f\left(k_{r-1}\right) \\
= & \left|f\left(k_{r}\right)-f\left(k_{r-1}\right)\right|=f\left(\left|k_{r}-k_{r-1}\right|\right) \\
= & f\left(\left|h_{r}\right|\right)=f\left(h_{r}\right) . \\
&
\end{aligned}
$$

Using (47) in (46), we have

$$
\begin{aligned}
& \frac{1}{f\left(h_{r}\right)} f\left(\left|\left\{k \in I_{r}:\left|x_{k}-l\right| \geq \varepsilon\right\}\right|\right) \\
& \quad \leq \frac{1}{\sum_{\substack{\left.I_{r, i}^{\prime} \subset I_{r} \\
1 \leq i \leq \nu\right)}} f\left(h_{r, i}^{\prime}\right)} \sum_{\substack{I_{r, i}^{\prime} \subset I_{r} \\
1 \leq i \leq \nu(r)}} f\left(h_{r, i}^{\prime}\right) t_{r, i},
\end{aligned}
$$

where $t_{r, i}=\left(f\left(h_{r, i}^{\prime}\right)\right)^{-1} f\left(\left|\left\{k \in I_{r, i}^{\prime}:\left|x_{k}-l\right| \geq \varepsilon\right\}\right|\right)$. Since the term on the right hand side of (48) is a regular weighted mean transformation of $\left(t_{r, i}\right)$, which tends to zero as $r \rightarrow \infty$, therefore the term on the right hand side of (48) also tends to zero as $r \rightarrow \infty$. Thus,

$$
\frac{1}{f\left(h_{r}\right)} f\left(\left|\left\{k \in I_{r}:\left|x_{k}-l\right| \geq \varepsilon\right\}\right|\right) \longrightarrow 0
$$

Our next result deals with the reverse inclusion.

Theorem 31. Let $f$ be an unbounded modulus and $\beta=\left(k_{m}^{\prime}\right)$ is a lacunary refinement of the lacunary sequence $\theta=\left(k_{r}\right)$. Let $I_{r}=\left(k_{r-1}, k_{r}\right], h_{r}=k_{r}-k_{r-1}, r=1,2,3, \ldots$, and $I_{m}^{\prime}=$ $\left(k_{m-1}^{\prime}, k_{m}^{\prime}\right], h_{m}^{\prime}=k_{m}^{\prime}-k_{m-1}^{\prime}, m=1,2,3, \ldots$. If there exists $\delta>0$ such that

$$
\frac{f\left(h_{m}^{\prime}\right)}{f\left(h_{r}\right)} \geq \delta \quad \text { for every } I_{m}^{\prime} \subset I_{r},
$$

then $x \in S_{\theta}^{f}$ implies $x \in S_{\beta}^{f}$.

Proof. For any $\epsilon>0$, and every $I_{m}^{\prime}$, we can find $I_{r}$ such that $I_{m}^{\prime} \subset I_{r}$; then we have

$$
\begin{aligned}
& \frac{1}{f\left(h_{m}^{\prime}\right)} f\left(\left|\left\{k \in I_{m}^{\prime}:\left|x_{k}-l\right| \geq \varepsilon\right\}\right|\right) \\
& \quad \leq \frac{1}{f\left(h_{m}^{\prime}\right)} f\left(\left|\left\{k \in I_{r}:\left|x_{k}-l\right| \geq \varepsilon\right\}\right|\right) \\
& \quad=\frac{f\left(h_{r}\right)}{f\left(h_{m}^{\prime}\right)} \frac{1}{f\left(h_{r}\right)} f\left(\left|\left\{k \in I_{r}:\left|x_{k}-l\right| \geq \varepsilon\right\}\right|\right) \\
& \quad \leq \frac{1}{\delta} \frac{1}{f\left(h_{r}\right)} f\left(\left|\left\{k \in I_{r}:\left|x_{k}-l\right| \geq \varepsilon\right\}\right|\right)
\end{aligned}
$$

from where it follows that $S_{\theta}^{f} \subset S_{\beta}^{f}$.

In the next theorem we deal with a more general situation.

Theorem 32. Let $f$ and $g$ be any two unbounded modulus functions such that $f(x) \leq g(x)$, for all $x \in[0, \infty)$, and $\beta=\left(k_{m}^{\prime}\right)$ is a lacunary refinement of the lacunary sequence $\theta=\left(k_{r}\right)$. Let $I_{r}=\left(k_{r-1}, k_{r}\right], h_{r}=k_{r}-k_{r-1}, r=1,2,3, \ldots$, and $I_{m}^{\prime}=\left(k_{m-1}^{\prime}, k_{m}^{\prime}\right], h_{m}^{\prime}=k_{m}^{\prime}-k_{m-1}^{\prime}, m=1,2,3, \ldots$. If there exists $0<\delta \leq 1$ such that

$$
\frac{f\left(h_{m}^{\prime}\right)}{g\left(h_{r}\right)} \geq \delta \quad \text { for every } I_{m}^{\prime} \subset I_{r},
$$

then $x \in S_{\theta}^{g}$ implies $x \in S_{\beta}^{f}$.

Proof. For any $\epsilon>0$, and every $I_{m}^{\prime}$, we can find $I_{r}$ such that $I_{m}^{\prime} \subset I_{r}$; then we have

$$
\begin{aligned}
& \frac{1}{f\left(h_{m}^{\prime}\right)} f\left(\left|\left\{k \in I_{m}^{\prime}:\left|x_{k}-l\right| \geq \varepsilon\right\}\right|\right) \\
& \quad \leq \frac{1}{f\left(h_{m}^{\prime}\right)} g\left(\left|\left\{k \in I_{m}^{\prime}:\left|x_{k}-l\right| \geq \varepsilon\right\}\right|\right) \\
& \quad \leq \frac{1}{f\left(h_{m}^{\prime}\right)} g\left(\left|\left\{k \in I_{r}:\left|x_{k}-l\right| \geq \varepsilon\right\}\right|\right) \\
& \quad=\frac{g\left(h_{r}\right)}{f\left(h_{m}^{\prime}\right)} \frac{1}{g\left(h_{r}\right)} g\left(\left|\left\{k \in I_{r}:\left|x_{k}-l\right| \geq \varepsilon\right\}\right|\right) \\
& \leq \frac{1}{\delta} \frac{1}{g\left(h_{r}\right)} g\left(\left|\left\{k \in I_{r}:\left|x_{k}-l\right| \geq \varepsilon\right\}\right|\right),
\end{aligned}
$$

from where it follows that $S_{\theta}^{g} \subset S_{\beta}^{f}$.

Hence $x \in S_{\theta}^{f}$.

as $r \longrightarrow \infty$. 
In the next theorem we show that the inclusion $S_{\theta}^{f} \subset S_{\beta}^{f}$ is possible even if none of $\theta$ and $\beta$ is refinement of the other.

Theorem 33. Let $f$ be an unbounded modulus such that

$$
|f(x)-f(y)|=f(|x-y|), \quad \forall x \geq 0, \quad y \geq 0 .
$$

Suppose $\beta=\left(k_{m}^{\prime}\right)$ and $\theta=\left(k_{r}\right)$ are two lacunary sequences. Let $I_{r}=\left(k_{r-1}, k_{r}\right], h_{r}=k_{r}-k_{r-1}, r=1,2,3, \ldots, I_{m}^{\prime}=\left(k_{m-1}^{\prime}, k_{m}^{\prime}\right]$, $h_{m}^{\prime}=k_{m}^{\prime}-k_{m-1}^{\prime}, m=1,2,3, \ldots$, and $I_{r m}=I_{r} \cap I_{m}^{\prime}, r, m=$ $1,2,3, \ldots$. If there exists $\delta>0$ such that

$$
\frac{f\left(\rho_{r m}\right)}{f\left(h_{r}\right)} \geq \delta \quad \text { for every } r, m=1,2,3, \ldots,
$$

provided $\rho_{r m}>0$, where $\rho_{r m}$ denotes the length of the interval $I_{r m}$, then $x \in S_{\theta}^{f}$ implies $S_{\beta}^{f}$.

The proof is similar to Theorem 2 of $\mathrm{Li}[14]$ and hence is omitted.

Remark 34. If the condition in Theorem 33 is replaced by $f\left(\rho_{r m}\right) / f\left(h_{m}^{\prime}\right) \geq \delta$ for every $r, m=1,2, \ldots$, provided $\rho_{r m}>0$, where $\rho_{r m}$ denotes the length of the interval $I_{r m}=I_{r} \cap$ $I_{m}^{\prime}, r, m=1,2,3, \ldots$, it can be seen that $x \in S_{\beta}^{f}$ implies $x \in S_{\theta}^{f}$.

Combining Remark 34 and Theorem 33, we get the following.

Theorem 35. Let $f$ be an unbounded modulus such that

$$
|f(x)-f(y)|=f(|x-y|), \quad \forall x \geq 0, \quad y \geq 0 .
$$

Suppose $\beta=\left(k_{m}^{\prime}\right)$ and $\theta=\left(k_{r}\right)$ are two lacunary sequences. Let $I_{r}=\left(k_{r-1}, k_{r}\right], h_{r}=k_{r}-k_{r-1}, r=1,2,3, \ldots, I_{m}^{\prime}=\left(k_{m-1}^{\prime}, k_{m}^{\prime}\right]$, $h_{m}^{\prime}=k_{m}^{\prime}-k_{m-1}^{\prime}, m=1,2,3, \ldots$, and $I_{r m}=I_{r} \cap I_{m}^{\prime}, r, m=$ $1,2,3, \ldots$. If there exists $\delta>0$ such that

$$
\frac{f\left(\rho_{r m}\right)}{f\left(h_{r}+h_{m}^{\prime}\right)} \geq \delta \quad \text { for every } r, m=1,2,3, \ldots,
$$

provided $\rho_{r m}>0$, where $\rho_{r m}$ denotes the length of the interval $I_{r m}$, then $S_{\theta}^{f}=S_{\beta}^{f}$.

\section{The $S_{\theta}^{f}$-Cauchy Criterion and a Tauberian Theorem}

We now introduce the $S_{\theta}^{f}$-analog of the Cauchy criterion, which turns out to be equivalent to the $S_{\theta}^{f}$-convergence.

Definition 36. Let $\theta=\left(k_{r}\right)$ be a lacunary sequence and let $f$ be an unbounded modulus. The sequence $x=\left(x_{k}\right)$ is said to be $f$-lacunary statistically Cauchy or $S_{\theta}^{f}$-Cauchy sequence if there is a subsequence $\left(x_{k^{\prime}(r)}\right)$ of $x$ such that $k^{\prime}(r) \in I_{r}$, for each $r, \lim _{r} x_{k^{\prime}(r)}=l$, and, for every $\epsilon>0$,

$$
\lim _{r \rightarrow \infty} \frac{1}{f\left(h_{r}\right)} f\left(\left|\left\{k \in I_{r}:\left|x_{k}-x_{k^{\prime}(r)}\right| \geq \varepsilon\right\}\right|\right)=0 .
$$

Theorem 37. The sequence $x$ is $S_{\theta}^{f}$-convergent if and only if $x$ is $S_{\theta}^{f}$-Cauchy sequence, where $f$ is an unbounded modulus and $\theta=\left(k_{r}\right)$ is a lacunary sequence.

The proof is similar to proof of Theorem 2 of Fridy and Orhan [13] and hence is omitted.

Corollary 38. Every $S_{\theta}^{f}$-convergent sequence has a convergent subsequence.

This result leads to the following Tauberian theorem. We state the following result, which can be established following the technique of Theorem 3 of Fridy and Orhan [13].

Theorem 39. If $S_{\theta}^{f}-\lim x_{k}=l$ and $\lim _{r \rightarrow \infty} h_{r} \max \left\{\left|\Delta x_{i}\right|:\right.$ $\left.i \in I_{r}\right\}=0$ then $\lim _{k} x_{k}=l$.

\section{Conflict of Interests}

The authors declare that there is no conflict of interests regarding the publication of this paper.

\section{References}

[1] A. Zygmund, Trigonometric Series, Cambridge University Press, London, UK, 2nd edition, 1979.

[2] H. Fast, "Sur la convergence statistique," Colloquium Mathematicum, vol. 2, pp. 241-244, 1951.

[3] H. Steinhaus, "Sur la convergence ordinaire et la convergence asymptotique," Colloquium Mathematicum, vol. 2, pp. 73-74, 1951.

[4] I. J. Schoenberg, "The integrability of certain functions and related summability methods," The American Mathematical Monthly, vol. 66, pp. 361-375, 1959.

[5] A. Aizpuru, M. C. Listán-García, and F. Rambla-Barreno, "Density by moduli and statistical convergence," Quaestiones Mathematicae, vol. 37, no. 4, pp. 525-530, 2014.

[6] V. K. Bhardwaj and S. Dhawan, " $f$-statistical convergence of order $\alpha$ and strong Cesàro summability of order $\alpha$ with respect to a modulus," Journal of Inequalities and Applications, 2015:332, 14 pages, 2015.

[7] H. Çakalli, "Lacunary statistical convergence in topological groups," Indian Journal of Pure and Applied Mathematics, vol. 26, no. 2, pp. 113-119, 1995.

[8] H. Çakalli, "A study on statistical convergence," Functional Analysis, Approximation and Computation, vol. 1, no. 2, pp. 1924, 2009.

[9] H. Çakalli and M. K. Khan, "Summability in topological spaces," Applied Mathematics Letters, vol. 24, no. 3, pp. 348-352, 2011.

[10] J. S. Connor, "The statistical and strong $p$-Cesàro convergence of sequences," Analysis, vol. 8, no. 1-2, pp. 47-63, 1988.

[11] J. Connor, "On strong matrix summability with respect to a modulus and statistical convergence," Canadian Mathematical Bulletin, vol. 32, no. 2, pp. 194-198, 1989.

[12] J. A. Fridy and C. Orhan, "Lacunary statistical convergence," Pacific Journal of Mathematics, vol. 160, no. 1, pp. 43-51, 1993.

[13] J. A. Fridy and C. Orhan, "Lacunary statistical summability", Journal of Mathematical Analysis and Applications, vol. 173, no. 2, pp. 497-504, 1993. 
[14] J. Li, "Lacunary statistical convergence and inclusion properties between lacunary methods," International Journal of Mathematics and Mathematical Sciences, vol. 23, no. 3, pp. 175-180, 2000.

[15] I. Niven and H. S. Zuckerman, An Introduction to the Theory of Numbers, John Wiley \& Sons, New York, NY, USA, 4th edition, 1980.

[16] H. Nakano, "Concave modulars," Journal of the Mathematical Society of Japan, vol. 5, pp. 29-49, 1953.

[17] W. H. Ruckle, "FK spaces in which the sequence of coordinate vectors is bounded," Canadian Journal of Mathematics, vol. 25, pp. 973-978, 1973.

[18] I. J. Maddox, "Inclusions between FK spaces and Kuttner's theorem," Mathematical Proceedings of the Cambridge Philosophical Society, vol. 101, no. 3, pp. 523-527, 1987.

[19] E. Öztürk and T. Bilgin, "Strongly summable sequence spaces defined by a modulus," Indian Journal of Pure and Applied Mathematics, vol. 25, no. 6, pp. 621-625, 1994.

[20] D. Ghosh and P. D. Srivastava, "On some vector valued sequence spaces defined using a modulus function," Indian Journal of Pure and Applied Mathematics, vol. 30, no. 8, pp. 819-826, 1999.

[21] V. K. Bhardwaj and N. Singh, "On some sequence spaces defined by a modulus," Indian Journal of Pure and Applied Mathematics, vol. 30, no. 8, pp. 809-817, 1999.

[22] V. K. Bhardwaj and N. Singh, "Some sequence spaces defined by $\left|\bar{N}, p_{n}\right|$ summability and a modulus function," Indian Journal of Pure and Applied Mathematics, vol. 32, no. 12, pp. 1789-1801, 2001.

[23] V. K. Bhardwaj and N. Singh, "Banach space valued sequence spaces defined by a modulus," Indian Journal of Pure and Applied Mathematics, vol. 32, no. 12, pp. 1869-1882, 2001.

[24] R. Çolak, "Lacunary strong convergence of difference sequence spaces with respect to a modulus function," Filomat, vol. 17, pp. 9-14, 2003.

[25] Y. Altin and M. Et, "Generalized difference sequence spaces defined by a modulus function in a locally convex space," Soochow Journal of Mathematics, vol. 31, no. 2, pp. 233-243, 2005.

[26] V. K. Bhardwaj, S. Dhawan, and S. Gupta, "Density by moduli and statistical boundedness," Abstract and Applied Analysis, vol. 2016, Article ID 2143018, 6 pages, 2016.

[27] A. R. Freedman, J. J. Sember, and M. Raphael, "Some Cesàrotype summability spaces," Proceedings of the London Mathematical Society, vol. 37, no. 3, pp. 508-520, 1978.

[28] I. J. Maddox, "Sequence spaces defined by a modulus," Mathematical Proceedings of the Cambridge Philosophical Society, vol. 100, no. 1, pp. 161-166, 1986.

[29] S. Pehlivan and B. Fisher, "On some sequence spaces," Indian Journal of Pure and Applied Mathematics, vol. 25, no. 10, pp. 1067-1071, 1994. 


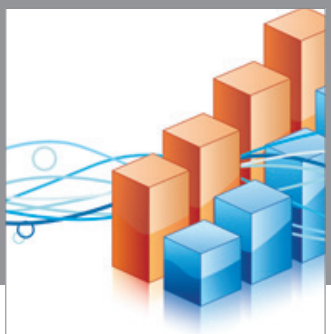

Advances in

Operations Research

vatem alat4

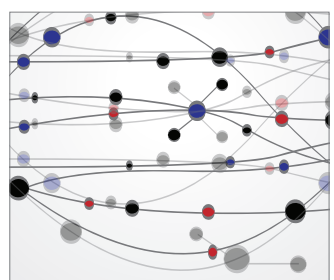

\section{The Scientific} World Journal
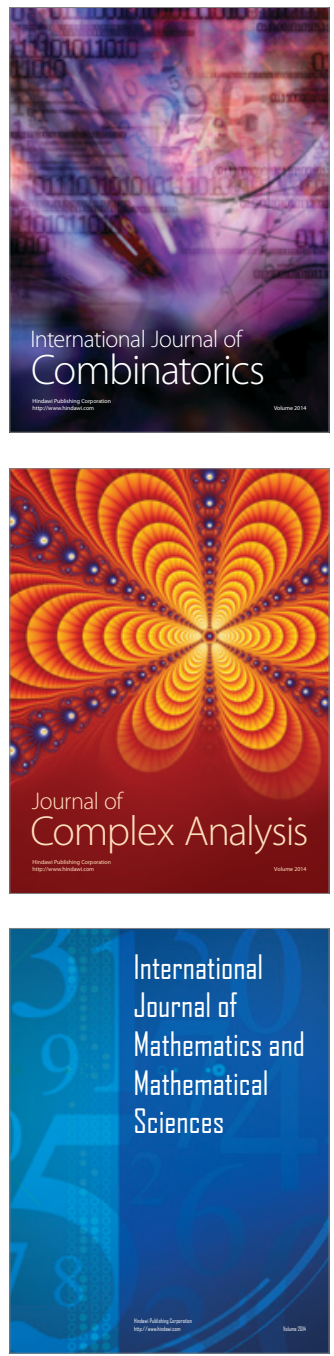
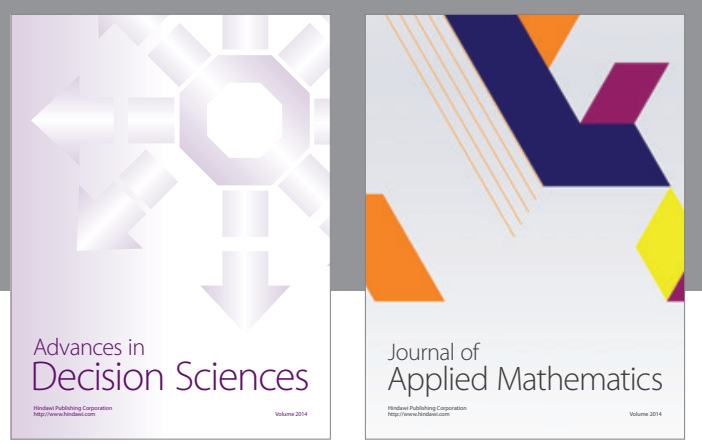

Algebra

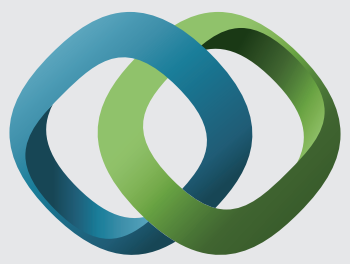

\section{Hindawi}

Submit your manuscripts at

http://www.hindawi.com
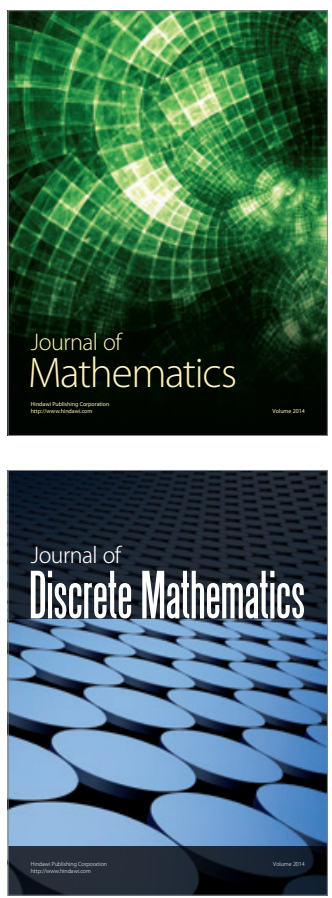

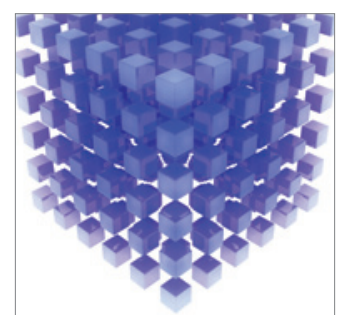

Mathematical Problems in Engineering
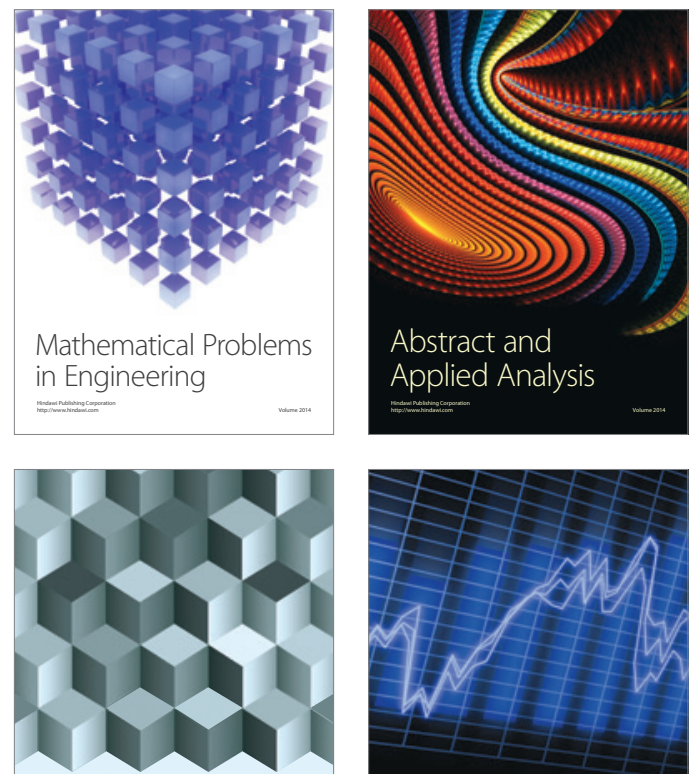

Journal of

Function Spaces

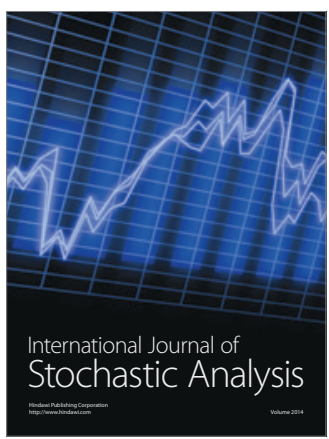

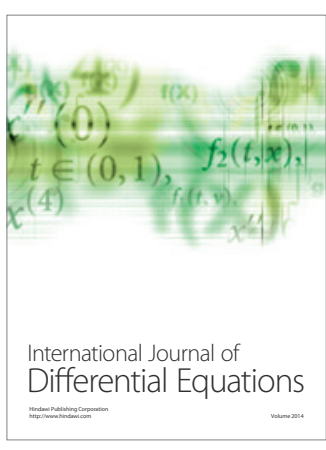
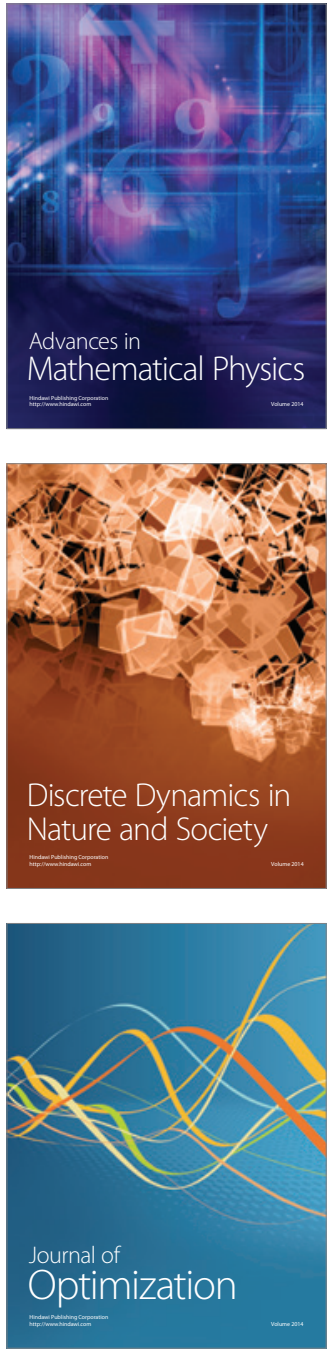